\title{
A Two Year Follow-up Study on the Success Rate of Dr. Poon's Metabolic Diet Program
}

\section{Pat Poon*}

Unit 101, 10 Royal Orchard Blvd Thornhill, Ontario, Canada

*Corresponding Author: Pat Poon, Unit 101, 10 Royal Orchard Blvd Thornhill, Ontario, Canada, Tel: 416-4614443; E-mail: doctor@poondiet.com

Received: 02 April 2018; Accepted: 06 April 2018; Published: 09 April 2018

\begin{abstract}
Lifestyle management is the treatment of choice to combat obesity and its related complications. Diet counseling is the main stage of lifestyle management. Exercise, stress management, sleeping hygiene, emotional eating and food addiction counseling are needed in addition to a good diet program to ensure that the diet is maintainable. The diet that was prescribed to the patients should be inexpensive, fulfill the nutritional need, and maintainable. A study was designed to determine the effectiveness of Dr. Poon's Metabolic Diet Program on weight reduction and weight maintenance. The result shows that when patients continue to attend the diet program, they were able to lose $10 \%$ of their initial weight on an average of 11 weeks and able to maintain the weight loss after two years. The average weight reduction after 2 years was $13.5 \%$.
\end{abstract}

Keywords: Combat obesity; Lifestyle management; Nutritional need

\section{Background}

In 2015, a pilot project was set up to assess the success rate of Dr. Poon's Metabolic Diet Program (DPMDP) [1]. A total of 384 overweight and obese patients were included in the study and their progress was followed for a period of 18 months. Sixty two percent of the patients were able to lose $10 \%$ of their original weight. The average amount of time to reach the $10 \%$ weight loss was 10 weeks. Among the patients who were able to lose $10 \%$ of their weight, $78 \%$ of them were able to lose additional weight, or maintained the $10 \%$ weight loss for up to 18 months, which was the length of that particular study. About $8 \%$ of the patients were able to achieve the normal BMI of 25. This is a follow up study of that pilot project. More patients were added to the study and were followed for a longer period of time. 
Most physicians believe that for obese patients, losing 5-10\% of their weight will improve their health [2-3]. Hence, some experts suggested that if a diet plan which can produce a $10 \%$ weight reduction in 1 year, and have dieters be able to maintain the weight loss, should be considered as a successful diet plan [4]. According to the US News in January 2018, the Weight Watcher's (WW) diet is voted as the best weight loss diet program [5]. According to the research done by Pinto et al, clients on the WW diet lost an average of 13 lbs in 48 weeks (less than 1 year) [6]. The average starting weight was 217.6 lbs. Therefore, patients on the WW diet plan lost an average of $6 \%$ of the initial weight in about 11 months. This is lower than the $10 \%$ weight loss criteria for a successful diet plan, as proposed by Rena Wing [4], one of the co-author of the WW study. Using the WW success rate as a guideline, any diet plan that can help an obese patient to reduce $6 \%$ of the original weight and kept it off for 1 year can be labeled as a successful diet. If a patient was 5' 5”, $200 \mathrm{lb}$ patient with an original body mass index (BMI) was 33.3 (a BMI of over 30 is considered obese) loses $6 \%$ of the initial weight (12lbs), the BMI will still be 31.3 which remains in the obesity zone. A successful weight loss diet should expect to produce better result than that. Another criterion of a good diet is that patients should be able to maintain for a long period of time. Some expect uses a period of one year as the standard of weight loss maintenance [4]. One of the goals of this study is to find out the patients that lost $10 \%$ of their original weight on the DPMDP, how many were able to maintain the $10 \%$ weight reduction for the next 2 years.

\section{Method}

A total of 590 new obese patients, from January 15, 2014 to March 15, 2014 were newly admitted to the DPMDP. Patients who did not return to the diet program for a follow up visit in the first month and patients with type 1 diabetes, end-stage renal failure and/or liver failure were excluded from this study.

All accepted patients were either obese (92\%) or overweight (8\%). All patients were suffering from disease related to their weight, such as diabetes, dyslipidemia, hypertension, arthritis, fatty liver, etc. The patient's weight and medical conditions were monitored for a period of 24 months, or until the patient dropped out of the program. Most patients receive lifestyle counseling in the clinics on a biweekly basis.

DPMDP is a lifestyle management program which includes diet, exercise, sleeping hygiene and stress management counseling. Optional group counseling on label reading, human nutrition and emotional eating is also provided. We have an online support group on Facebook to provide patient-to-patient support, to provide resources such as recipes, and to facilitate local patient support meetings. There are more than 4000 patients actively participating on this Facebook group.

The DPMDP consists of 3 phases, and is not a "how-much-to-eat" but a "what-to-eat" type of diet plan. It also does not require the patients to count calories, or measure the amount of food consumed. Patients are told to eat until they are full using a set of dietary guidelines, and the patients can eat the allowed food items at any time of the day. The main objective of the diet is to consume mostly proteins (for the essential amino acids), good fats (for the essential fatty acids), and high fiber vegetables, while limiting the consumption of sugar, starch, saturated fat, trans fat, 
alcohol and sodium. In the ideal scenario, the patients should be losing fat and excess water, while preserving the lean muscle mass. Phase One of the DPMDP has the lowest amount of sugar and starch allowed, while small amounts of sugar and starch are re-introduced when the patient progresses to Phase Two and Phase Three of the DPMDP. Phase One consists of all the essential nutrients that human needs for good health. There is no maximum limit for how long the patients remained on Phase One; however, a minimum of two weeks on Phase One is required. Some carbohydrates are re-introduced to the diet in Phase Two and Phase Three, to provide personal enjoyment and to satisfy potential food cravings, but not for their nutritional benefit.

Tanita Body Composition Analyzers are used in all the clinics to assess the patient's weight and body composition. All data are electronically recorded. There are no follow-ups with the patients who discontinued the program on their own accord.

\section{Results}

The total number of patients was 590, with 144 males (24.4\%) and 446 females (75.6\%), and an average age of 48.5 years old. Most patients had tried other diet programs before joining the DPMDP. The average weight of the patients before starting the DPMDP was $224.3 \mathrm{lbs}$, with an average BMI of 36.9. Figure 1 shows the average weight of patients who were still on the DPMDP during the 2 year period. The average weight of these was 199.6 lbs after 6 months, 201.5 lbs after 12 months, 204.0 lbs after 1 months and 204.8 lbs after 24 months. Figure 2 shows the average weight reduction was $12.7 \%$ after 6 months, $11.9 \%$ after 12 months, $10.8 \%$ after 18 months and $11.3 \%$ after 24 months. The summary is shown in Table 1.

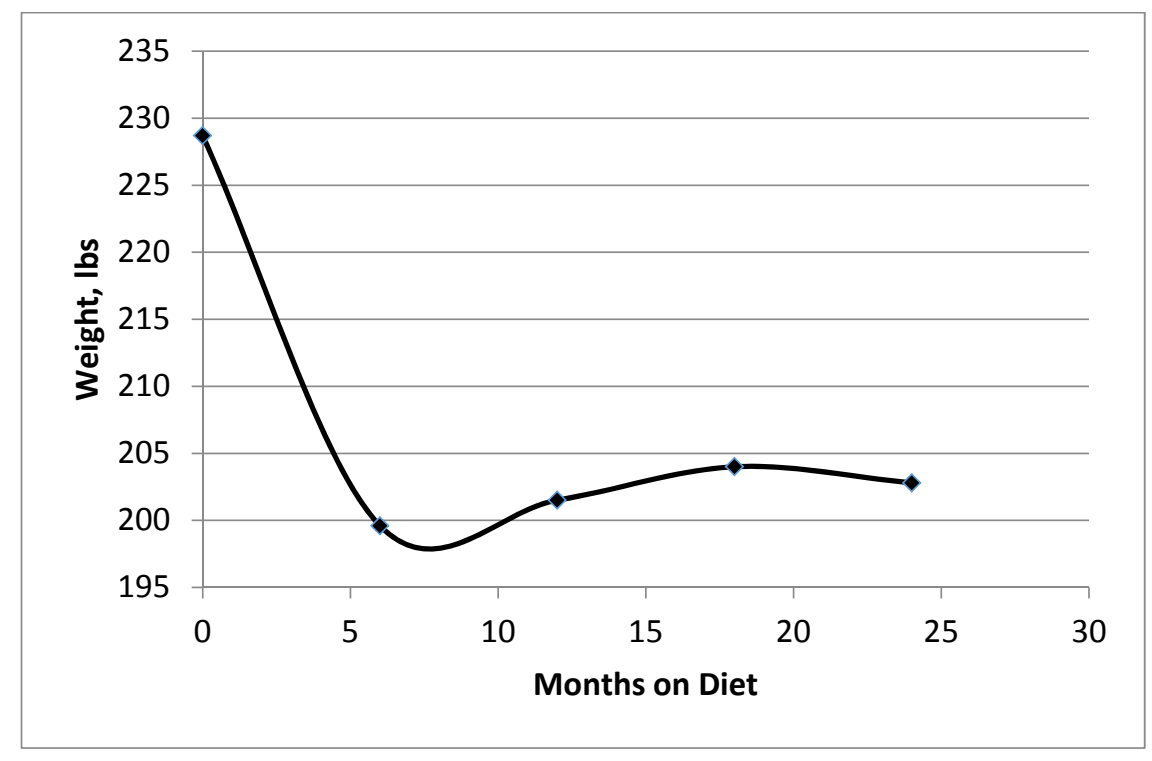

Figure 1: Average Weight of Patients during the Course of Dieting. 


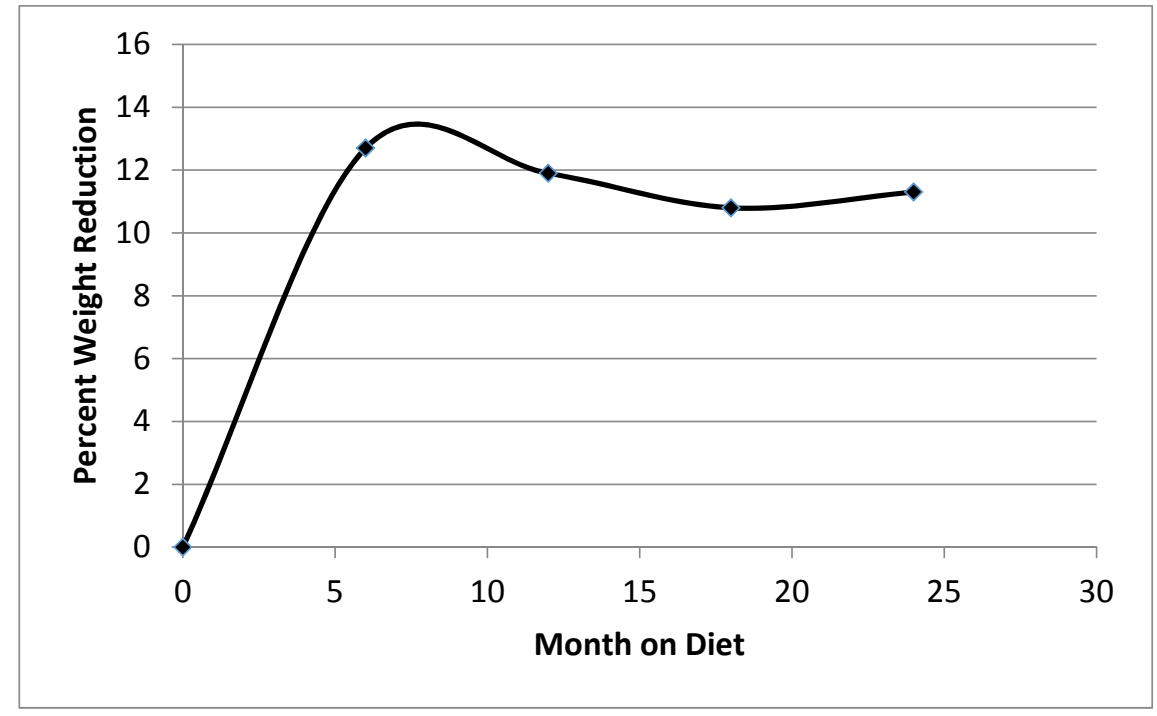

Figure 2: Average Percentage of Weight Reduction during the Course of Dieting.

\begin{tabular}{|c|c|c|c|}
\hline Months on the diet & $\begin{array}{c}\text { Number of patients } \\
\text { remaining }\end{array}$ & Average weight & \% weight reduction \\
\hline 0 & 590 & 228.7 & 0 \\
\hline 6 & 353 & 199.6 & 12.7 \\
\hline 12 & 246 & 201.5 & 11.9 \\
\hline 18 & 195 & 204.0 & 10.8 \\
\hline 24 & 71 & 202.8 & 11.3 \\
\hline
\end{tabular}

Table 1: Summary of the average weight and percent weight loss during the course of the diet.

480 patients (81\%) achieved a weight reduction of $5 \%$ or more of their original weight, during their time on the DPMDP. 353 patients (60\%) achieved a weight loss of $10 \%$ of their original weight. Most of the patients achieving this $10 \%$ weight loss goal within 10 to 20 weeks, with a mean time of 11 weeks, when $2.5 \%$ of the outliers from both high and low ends were eliminated from the calculation (Figure 3). For all the patients who lost $10 \%$ of their original weight, $62 \%$ of them still able maintain the $10 \%$ weight loss when they stay with the DPMDP. $38 \%$ of the patients who lost $10 \%$ of the initial weight regained some, or all, of their weight.

After 24 months, 71 patients (26\% male and 74\% female) continued to attend the DPMDP follow-up visits. These percentages are similar to the original set of patients before dieting. The average weight of this group of 71 patients was 234.4 lbs before starting the DPMDP, and their average weight after 24 months was 202.8 lbs. This represents an average of 13.5\% weight reduction after 24 months on the diet plan. 39 patients (6.6\%) achieved a normal BMI 
of 25 within 24 months. There were no incidents of diet-related medical complication leading to discontinuation of the DPMDP.

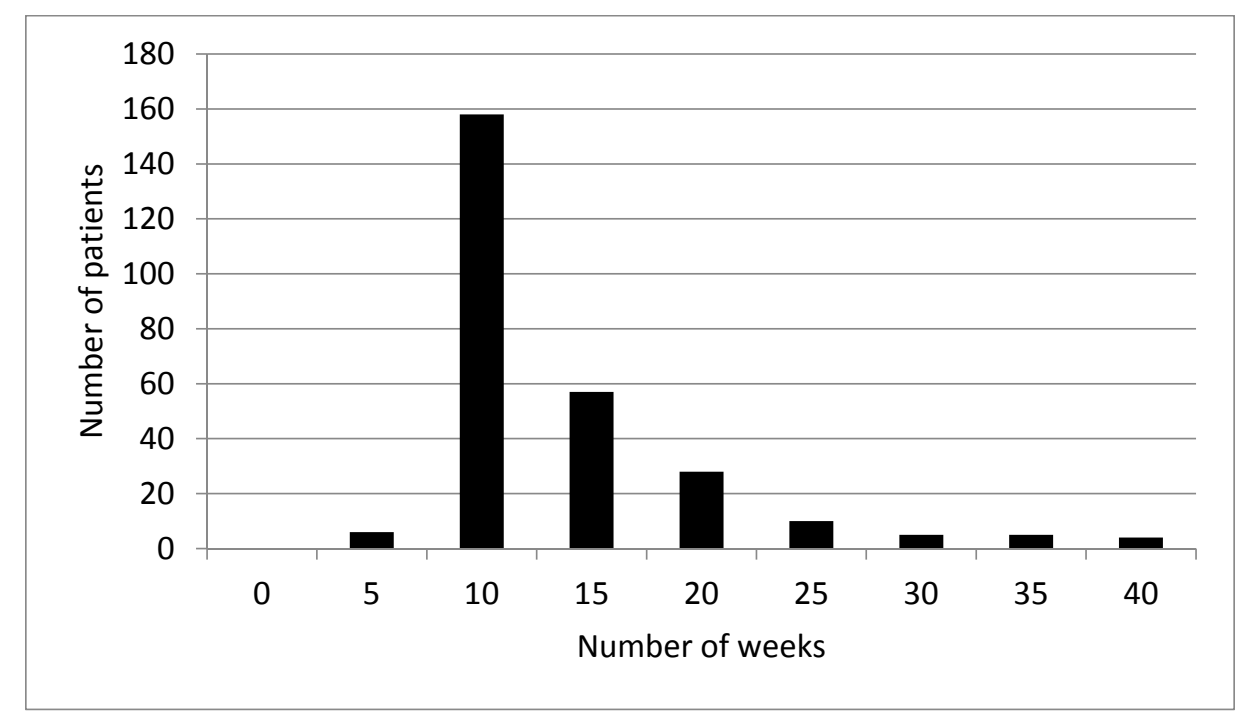

Figure 3: Number of weeks to achieve $10 \%$ weight reduction.

\section{Discussion}

Some studies consider $5 \%$ weight loss as a sign of a successful weight loss. $81 \%$ percent of DPMDP patients were able to achieve a 5\% weight reduction. Instead of using a 5\% weight reduction as the threshold for weight loss success, the DPMDP uses a 10\% weight loss threshold. Experts believe that with a $10 \%$ weight reduction, abnormal metabolic parameters of patients will improve. Results show that most patients on DPMDP lost $10 \%$ of their initial weight within 11 weeks (Figure 1). More important though, is how many patients maintained the 10\% weight reduction with time. Figure 2 shows that after 8 months on the diet, the average amount of weight lost peaked at about $13 \%$ of patient's initial weight. For the 24 month period, patients still on the DPMDP were able to keep their average weight reduction above the $10 \%$ weight loss threshold. Previous studies show that most diet programs help patients lose weight, but most of the patients regain most of their weight loss after 6 to 12 months [7, 8]. The average starting weight of our 590 patients was 228.7 lbs, with the average weight dropping down to 219.6 lbs after 6 months on the DPMDP (Table 1). This represented a 29 lbs (12.7\%) reduction in 6 months. After 2 years, the average weight was $202.8 \mathrm{lbs}$, which means that on an average, patients only regained $3 \mathrm{lbs}$ after 18 months.

The objective of this study is to assess the long-term success rate of the DPMDP; hence, it is important to analyze those patients who remained on the DPMDP long-term. There are 71 out of the total 590 patients who still attended the DPMDP after 2 years. These patients were able to lose weight and maintain a 13.5\% weight loss after 2 years. The result is better than the $11.3 \%$ weight reduction of the whole group. The result indicates that most patients can lose significant weight on this diet, and that the weight reduction is maintainable. Attending the DPMDP clinics on a consistent basis helped with weight loss and weight maintenance. 
A weight loss of $10 \%$ of the original is a critical goal, but the ultimate goal is for patients to achieve a healthy BMI of 25 or less. $6.6 \%$ of all the patients in this study were able to achieve a BMI of 25. These patients were encouraged to return to the DPMDP clinic every 3 to 6 months, to weigh-in, and to discuss any problems that may have arisen during the maintenance period. This helps remind patients that they have not completed the DPMDP, but rather, that they are still in the DPMDP maintenance phase, and are being monitored by their doctors.

These following comments are examples of comments that often come from patients before starting the DPMDP: "Diet A worked, but I did not continue the diet and regained my weight." "Diet B worked, but I had to give up the diet because I got pregnant." "Diet C worked, but it was too expensive for me to continue." "Diet D worked, but I cannot stay hungry forever." "Diet E worked, but it was too hard to eat replacement bars and shakes for the rest of my life.” Fad diets are defined as diet plans that are unsustainable. Fad diets lead to yo-yo dieting, which can lower a patient's metabolic rate. Any good diet plan must be maintainable.

According to National Institutes of Health (NIH) [9], safe and successful weight-loss programs should include:

- "Behavioral treatment, also called lifestyle counseling that can teach you how to develop and stick with healthier eating and physical activity habits" - DPMDP provides counseling on exercise and emotional eating.

- "Information about getting enough sleep, managing stress, and the benefits and drawbacks of weight-loss medicines” - DPMDP provides counseling on sleep hygiene, stress management and does not require patients to take medications or supplements.

- "Ongoing feedback, monitoring, and support throughout the program, either in person, by phone, online, or through a combination of these approaches" - DPMDP provides regular feedback to patient every two weeks. The DPMDP sponsored a patient-run Facebook Support Group that provides free recipes and open discussion on the DPMDP. The discussions on the Facebook Group are monitored by the DPMDP, to ensure accuracy.

- "Slow and steady weight-loss goals"- patients on the DPMDP usually achieve a 10\% weight loss every 8 to 10 weeks. Weight loss should not hit a plateau as long as the patient continues to follow the DPMDP.

- "A plan for keeping the weight off, including goal setting, self-checks" - DPMDP provides patients with a maintenance program, and patients on the maintenance program are seen every 3-6 months to monitor their weight, and to receive ongoing lifestyle management counseling. This study demonstrates that this diet is maintainable.

DPMDP is very close to, if not entirely, satisfies with the NIH's definition of a "safe and successful weight-loss program". The DPMDP also exceeded the $10 \%$ weight loss and the 1-year weight loss maintenance thresholds, expected of a successful weight loss plan. Patients who stayed with the DPMDP lost more than $10 \%$ of their initial weight, and kept it off for 2 years. 
We will continue to monitor this group of patients, and will enroll more patients to this study, to increase the power of the study. We will also study the effect of a $10 \%$ weight reduction on the metabolic syndrome symptoms in this group of patients. Not all patients that lost $10 \%$ of their initial weight could maintain, or increase, their overall weight loss. A possible explanation for this group's inability to do so is food addiction. There may be a potential use for the combination prescription medication of naltrexone plus bupropion, to help this group of patients maintain their weight loss and/or further weight reduction.

\section{References}

1. Poon P. A Pilot Project to Assess the Success Rate of Dr. Poon's Metabolic Diet Program. Obes Open Access 2 (2015): 114.

2. National Heart, Lung, and Blood Institute. Clinical guidelines on the identification, evaluation, and treatment of overweight and obese in adults: The evidence report. Obes Res 6 (suppl) (1988): 51S-201S.

3. Jensen MD, Ryan DH, Apovian CM, et al. Guidelines for the management of overweight and obesity in adults. Obesity 22 (2013): S41-S410.

4. Wing RR, Hill JO. Successful weight loss maintenance. Annu Rev Nutr 21 (2001): 323-341.

5. https://health.usnews.com/wellness/food/articles/how-us-news-ranks-best-diets.

6. Pinto AM, Fave JL, Hoffmann DA, et al. Combining Behavioral Weight Loss Treatment and a Commercial Program: A Randomized Clinical Trial. Obesity 21 (2013): 4.

7. Kraschnewski JL, Boan J, Esposito J, et al. Long-term weight loss maintenance in the United States. Int J Obes (Lond) 4 (2010): 1644-1654.

8. Weiss EC, Galuska DA, Kettel Khan L, et al. Weight regain in U.S. adults who experienced substantial weight loss, 1999-2002. Am J Prev Med 33 (2007): 34-40.

9. https://www.niddk.nih.gov/health-information/weight-management/choosing-a-safe-successful-weightloss-program.

Citation: Pat Poon. A Two Year Follow-up Study on the Success Rate of Dr. Poon's Metabolic Diet Program. Archives of Clinical and Biomedical Research 2 (2018): 078-084.

This article is an open access article distributed under the terms and conditions of the Creative Commons Attribution (CC-BY) license 4.0 